\title{
Avaliação da atividade antioxidante e identificação dos ácidos fenólicos presentes no bagaço de maçã cv. Gala
}

\author{
Evaluation of antioxidant activity and identification of phenolic \\ acids present in the pomace of Gala variety apples \\ Marcia SOARES ${ }^{1}$, Lucas WELTER ${ }^{1}$, Luciano GONZAGA ${ }^{1}$, Alessandro LIMA ${ }^{2}$, \\ Jorge MANCINI-FILHO ${ }^{2 *}$, Roseane FETT ${ }^{1}$
}

\section{Resumo}

No processamento da maçã para obtenção do suco, ocorre a formação de uma elevada quantidade de resíduo, representado pelas cascas e sementes, no qual são encontradas diversas substâncias, como os compostos fenólicos, aos quais são atribuídas as propriedades antioxidantes. Este estudo teve como objetivo avaliar o conteúdo de compostos fenólicos totais e a atividade antioxidante in vitro de extratos e frações de ácidos fenólicos livres, esterificadas solúveis e esterificadas insolúveis, obtidos por diferentes solventes, a partir do resíduo da maçã cv. Gala pelos métodos DPPH (2,2-difenil-1-picrilhidrazilo) e ABTS [2,2'-azino-bis (3-etilbenzotiazolin) 6-ácido sulfônico], além da identificação dos ácidos fenólicos por cromatografia gasosa. O sistema acetona $75 \%$ (v/v) em água foi mais eficaz na extração dos compostos fenólicos totais.

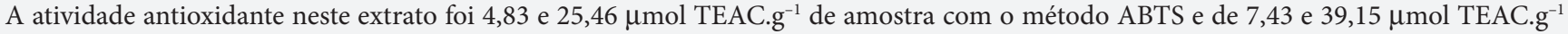
pelo método DPPH, expressos em base úmida e seca, respectivamente. O teor de flavanóis foi 11,05 e $58,20 \mathrm{mg}^{100} \mathrm{~g}^{-1} \mathrm{em}$ base úmida e seca, respectivamente. Houve uma correlação positiva entre o conteúdo de fenólicos totais e atividade antioxidante $\left(r^{2}=0,9283\right)$. Foram identificados os ácidos fenólicos salícilico, gálico, propilgalato e sináptico nas três frações avaliadas, o primeiro estando presente em maior concentração em todas as frações estudadas. Os resultados indicam o potencial antioxidante do bagaço de maçã.

Palavras-chave: bagaço de maçã cv. Gala; atividade antioxidante; ácidos fenólicos.

\begin{abstract}
Apple processing to obtain juice produces a great amount of discarded material constituted by peel and seed. Several substances, including phenolic compounds, to which antioxidant activity is attributed, are found in these fractions. This work aimed at evaluating total phenolic compounds and in vitro antioxidant activity of extracts and phenolic fractions (free, soluble esterified and insoluble esterified) of apple, cv. Gala, residues. The methodologies used were DPPH (2,2-diphenyl-1-picrylhydrazyl) and ABTS [2,2'-azino-bis(3-ethylbenzothiazoline)6 -sulfonic acid] to evaluate antioxidant activity and gas chromatography to determine phenolic acids. Extraction with $75 \%$ acetone (v/v) in water was the most efficient to obtain total phenolic compounds. The antioxidant activity in this extract was 4.83 and $25.46 \mu$ mol TEAC.g ${ }^{-1}$ by the ABTS method and 7.43 and $39.15 \mu$ mol TEAC. $\mathrm{g}^{-1}$ by the DPPH method for wet and dry samples, respectively. Flavanol contents in the same samples were 11.05 and $58.20 \mathrm{mg} .100 \mathrm{~g}^{-1}$ for wet and dry samples, respectively. A positive correlation between total phenolics and antioxidant activity $\left(\mathrm{r}^{2}=0.9283\right)$ was observed. In the three fractions used, the following phenolic acids were identified: salicylic, gallic, propyl gallate and synaptic acid, being the first present in the highest amount in all the fractions studied. The results obtained showed the antioxidant potential of apple pomace.
\end{abstract}

Keywords: Gala variety apple pomace; antioxidant activity; phenolic acids.

\section{Introdução}

O crescente interesse pelos antioxidantes naturais de extratos de plantas é devido à sua baixa toxicidade em relação aos antioxidantes sintéticos. Extratos de frutas, vegetais, cereais e seus subprodutos industriais são ricos em antioxidantes, em ácido ascórbico, tocoferóis, carotenóides e em compostos fenólicos e têm demonstrado eficaz atividade antioxidante em sistemas modelos (WOLFE; WU; LIU, 2003; MANACH et al., 2004).

Os compostos fenólicos são formados no metabolismo secundário dos vegetais e possuem funções de defesa contra o ataque de pragas. Já em animais e humanos tem-se observado que são capazes de reagir com radicais livres, formando radicais estáveis. Esse poder de neutralização das estruturas radicalares dos compostos fenólicos é devido à sua estrutura química formada por pelo menos um anel aromático com grupamentos hidroxilas (GIADA; MANCINI-FILHO, 2006).

Além da sua atividade antioxidante direta, pesquisas recentes têm destacado múltiplas funções e mecanismos importantes relacionados à habilidade dos compostos fenólicos de se ligarem

Recebido para publicação em $7 / 10 / 2007$

Aceito para publicação em 7/4/2008 (002911)

${ }^{1}$ Programa de Pós-Graduação em Ciência dos Alimentos, Ciência e Tecnologia de Alimentos - CAL, Centro de Ciências Agrárias - CCA,

Universidade Federal de Santa Catarina - UFSC, Rod. Admar Gonzaga, 1346, Itacorubi, CP 476, CEP 88934-001, Florianópolis - SC, Brasil

${ }^{2}$ Departamento de Alimentos e Nutrição Experimental, Faculdade de Ciências Farmacêuticas, Universidade de São Paulo - USP, Av. Prof. Lineu Prestes, 580, Bloco 14, CEP 05508-900, São Paulo-SP, Brasil,E-mail: jmancini@usp.br

${ }^{*}$ A quem a correspondência deve ser enviada 
a receptores celulares e transportadores de membranas e influenciarem a expressão gênica, a sinalização e a adesão celular (MANACH; MAZUR; SCALBERT, 2005).

São descritas várias funções fisiológicas para os compostos fenólicos presentes na maçã, incluindo atividade antialergênica in vivo e atividade inibitória in vivo e in vitro de algumas enzimas e receptores celulares (SHOJI; AKAZOME; IKEDA, 2004). A maçã apresenta elevado teor de fitonutrientes, tais como flavonóides, polifenóis e ácidos fenólicos, encontrados na polpa e principalmente na casca, portanto, fornece os benefícios antioxidantes atuando na redução do risco de doenças cardiovasculares, câncer entre outras (TSAU et al. 2005).

Atualmente existe uma série de métodos in vitro para avaliação da atividade antioxidante de extratos vegetais, tendo em vista a grande variedade de compostos com propriedades antioxidantes, além da complexidade quanto ao seu modo de combater os distintos radicais livres. Dentre os métodos descritos, os ensaios de captura de radicais livres DPPH (2,2-difenil1-picrilhidrazina) e ABTS [2,2'-azino-bis(3-etilbenzotiazolin) 6-ácido sulfônico] utilizam espécies radicalares estáveis e a detecção do ponto final se realiza geralmente por absorvância, sendo muito empregados na determinação da atividade antioxidante de alimentos, bebidas e de plasma (LU; FOO, 2000).

O bagaço de maçã, resíduo industrial oriundo da produção de suco, é constituído pela mistura heterogênea de casca, semente, cálice, haste e polpa (LOMMEN et al., 2000; JEWELL; CUMINGS, 1984). Segundo Lu e Foo (2000) e Pesche et al. (2005), os compostos bioativos da fruta in natura permanecem na biomassa fresca, mesmo após o processo de produção do suco. Portanto são relevantes estudos que avaliem estes materiais utilizados para descarte pela indústria alimentícia, tendo em vista que possivelmente contenham além de outros constituintes, compostos com propriedades antioxidantes. Neste sentido, o objetivo deste estudo foi avaliar a atividade antioxidante do extrato de bagaço de maçã utilizando diferentes sistemas solventes, assim como determinar o conteúdo de flavanóis e a composição em ácidos fenólicos.

\section{Material e métodos}

\subsection{Amostras}

As amostras de bagaço de maçã cv. Gala safra 2005/2006, obtidas após a etapa de prensagem, foram fornecidas pela Indústria Fischer Sucos (Videira-SC). As amostras foram congeladas em freezer doméstico, à temperatura de $-18 \pm 2{ }^{\circ} \mathrm{C}$, até serem analisadas. Um pré-tratamento foi realizado em estufa à temperatura de $80 \pm 2{ }^{\circ} \mathrm{C}$ por 10 minutos, a fim de inativar as enzimas polifenoloxidase (PPO) e peroxidase (POD) segundo metodologia previamente descrita por Valderrama, Maranfoni e Clemente (2001), que descrevem a inativação destas enzimas nesta temperatura. Em seguida, as amostras foram secas a $45 \pm 2{ }^{\circ} \mathrm{C}$ por 4 horas, trituradas em moinho de bancada (IKA A11 Basic) e padronizadas em granulometria de 60 mesch $(0,25 \mathrm{~mm})$. Até a obtenção dos extratos, as amostras foram armazenadas em embalagens de polietileno $\mathrm{a}-18 \pm 2{ }^{\circ} \mathrm{C}$.
Determinou-se a umidade inicial e final dos bagaços de maçã (AOAC, 1995) para padronização das amostras.

\subsection{Preparação dos extratos}

O resíduo seco do bagaço foi submetido à extração com diferentes sistemas solventes para determinar a efetividade do tipo de solvente na extração dos compostos fenólicos totais do bagaço de maçã (YILMAZ; TOLEDO, 2006). Os extratos foram preparados com 3,0 g de material seco, utilizando como solventes extratores, as seguintes soluções aquosas: etanol, metanol e acetona a 0, 25, 50, 75 e 100\% (v/v), sob agitação e ao abrigo total da luz, durante duas horas. Posteriormente os extratos foram filtrados em papel filtro Whatman $n^{\circ} 1$ e transferidos para balão volumétrico, com volume final ajustado para $50 \mathrm{~mL}$. O rendimento da extração foi calculado e seu resultado (\%) expresso em peso seco. Para determinação do teor de flavanóis e da atividade antioxidante, os extratos foram preparados com material seco a $45^{\circ} \mathrm{C}(3,0 \mathrm{~g})$ e material fresco $(5,0 \mathrm{~g})$, utilizando como solvente acetona $75 \%(\mathrm{v} / \mathrm{v})$.

\subsection{Determinação de polifenóis totais (PT)}

O conteúdo de Polifenóis Totais foi determinado pelo método espectrofotométrico desenvolvido por Folin-Ciocateau (ROSSI; SINGLETON, 1965). Preparou-se uma curva padrão com ácido gálico em concentração variando de 50 a 500 mg.L $\mathrm{L}^{-1}$, e os resultados foram expressos em mg equivalente de ácido gálico $100 . \mathrm{g}^{-1}$ de amostra (base seca). As análises foram realizadas em triplicata.

\subsection{Determinação de flavanóis totais}

A determinação de flavanóis totais foi realizada aplicandose o método DMACA ( $p$-dimetilaminocinmaldeído), descrito por Arnous, Marcus e Kefalas (2002). A concentração total de flavanóis foi estimada com base na curva padrão, preparada com solução de catequina (1-16 mg de catequina/L). Os resultados foram expressos em mg equivalente à catequina $100 \mathrm{~g}^{-1}$ de amostra.

\subsection{Atividade antioxidante - método ABTS (2,2'-azino-bis (3-etilbenzotiazolin) 6-ácido sulfônico)}

O método ABTS utilizado foi descrito por Re et al. (1999) e modificado por Kuskoski et al. (2004). A absorbância foi medida em espectrofotômetro modelo Hewlett-Packard 8452 A, no tempo de 7 minutos após a adição da amostra. Os resultados foram expressos em TEAC (atividade antioxidante equivalente ao Trolox (6-hidroxi-2,5,7,8-tetrametilcromo-2-ácido carboxílico)) em $\mu$ mol TEAC.g ${ }^{-1}$ de amostra, e em VCEAC (atividade antioxidante equivalente à vitamina $\mathrm{C}$, em mg VCEAC. $100 \mathrm{~g}^{-1}$ de amostra).

\subsection{Atividade antioxidante - método DPPH (2,2-difenil-1-picrilhidrazila)}

A capacidade antioxidante foi determinada também pela redução do radical estável DPPH através da ação dos antioxidantes presentes na amostra, de acordo com Brand-Willams, 
Cuvelier e Berset (1995), com modificações de Kim et al. (2002). A medida de absorbância do radical antes de adicionar a amostra $\left(A_{0}\right)$ e depois de 30 minutos de reação $\left(A_{f}\right)$ é realizada no comprimento de onda de $517 \mathrm{~nm}$. Os resultados são expressos em TEAC ( $\mu$ mol TEAC..$^{-1}$ de amostra).

\subsection{Identificação das frações de ácidos fenólicos do bagaço da maçã}

A obtenção de frações de compostos fenólicos presentes no bagaço de maçã foi realizada pelo método descrito por Krygier, Sosulski e Hogge (1982), com algumas modificações (SOTERO, 2002). A identificação dos ácidos fenólicos foi baseada nos tempos de retenção dos ácidos fenólicos padrões e realizada em cromatógrafo Shimadzu, modelo GC 17A, equipado com detector de ionização de chama. As condições cromatográficas seguiram os procedimentos descritos por Dabrowski e Sosulski (1984), com modificações propostas por Moreira e Mancini Filho (2003). Foi utilizada uma coluna semipolar DB5 (J \& W $W^{\circledast}$ ), medindo $30 \mathrm{~m} \times 0,25 \mathrm{~mm}$. As condições de operação foram as seguintes: temperatura inicial da coluna a $150{ }^{\circ} \mathrm{C}$, isotérmica por três minutos; rampa de 150 para $300{ }^{\circ} \mathrm{C}$, aumentando $5{ }^{\circ} \mathrm{C} /$ minuto; isotérmica a $300{ }^{\circ} \mathrm{C}$ por três minutos. A temperatura da câmara de injeção foi de $250^{\circ} \mathrm{C}$ e a do detector foi de $300{ }^{\circ} \mathrm{C}$. Uma solução padrão foi preparada diluindo-se os 18 ácidos fenólicos em metanol, e uma alíquota desta solução foi adicionada ao padrão interno (éster metílico do ácido heptadecanóico) antes da injeção no cromatógrafo.

\subsection{Análise estatística}

Os resultados foram expressos na forma de média \pm desvio padrão $(n=3)$. Para análise estatística dos resultados, utilizou-se o programa Statistica ${ }^{\circledR} 6.0$ da StatSoft (Tulsa, EUA). Foram utilizados análise de variância (ANOVA) e o teste de Tukey para identificar diferenças significativas entre as médias $(\mathrm{p}<0,05)$.

\section{Resultados e discussão}

\subsection{Determinação de fenólicos totais}

Os compostos fenólicos são os maiores responsáveis pela atividade antioxidante em frutos (HEIM; TAGLIAFERRO; BOBYLIA, 2002). Para que os compostos fenólicos sejam considerados antioxidantes e possam exercer seu papel biológico é necessário que, em baixas concentrações, sejam capazes de impedir, retardar ou prevenir a auto-oxidação ou oxidação mediada por radicais livres e que o produto formado após a reação seja estável (RICE-EVANS, 1996). Os resultados obtidos (Figura 1) demonstram que, dos diferentes solventes utilizados na extração dos fenólicos totais do bagaço de maçã da cv. Gala a acetona foi a que apresentou maior eficiência de extração.

A extração com acetona nas concentrações de 75\% e 100\% (v/v) apresentou maior conteúdo de compostos fenólicos $\left(467,24 \pm 7,20\right.$ mg GAE. $100 \mathrm{~g}^{-1}$ e 522,74 $\pm 4,02$ mg GAE. $100 \mathrm{~g}^{-1}$, base seca). Não houve diferença significativa entre as concentrações de $75 \%$ e $100 \%(\mathrm{v} / \mathrm{v})$ na variedade estudada $(\mathrm{p}<0,05)$. Esses resultados estão de acordo com o estudo feito por Yilmaz

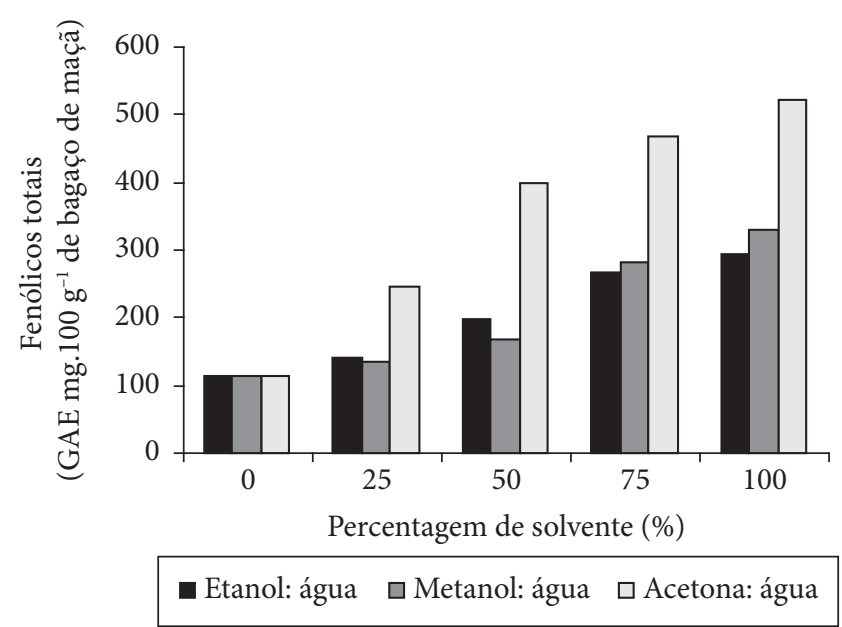

Figura 1. Fenólicos totais dos extratos obtidos do bagaço de maçã cv. Gala safra 2006 em diferentes sistemas de solventes (base seca).

e Toledo (2006), em cujo relatório misturas aquosas de acetona a $50 \%$ e $75 \%$ (v/v) foram mais eficientes na extração de constituintes fenólicos em semente de uva que os sistemas etanol $60 \%$ e metanol 70\% (v/v). Em pesquisa desenvolvida por Eberhardt, Lee e Liu (2000) com maçã cv. Red Delicious, utilizando como solvente extrator a acetona $80 \%$, foram obtidos teores de compostos fenólicos de 290,2 \pm 4,2 e 219,8 \pm 1,8 mg GAE. $100 \mathrm{~g}^{-1} \mathrm{em}$ base seca, em maçãs com e sem pele, respectivamente.

De acordo com os resultados apresentados na Figura 1, o conteúdo de fenólicos totais que permanece no bagaço de maçã é elevado (134,45 a 522,74 mg GAE. $100 \mathrm{~g}^{-1}$ em base seca), quando comparado com outros frutos e bagas. Stratil, Klejdus e Kubán (2007) relataram um conteúdo fenólico na maçã cv. Golden Delicious de 14,9 mg GAE.g ${ }^{-1}$ em base seca) e Kim, Jeong e Lee (2003) encontraram valores de 118,0 mg GAE.100 g-1 (base seca) em maçã Gala e 174 a 375 mg GAE. $100 \mathrm{~g}^{-1}$ em diversas variedades de acerola. Segundo Sun et al. (2002), na maçã fresca, o conteúdo de fenólicos totais é em média de 296,3 \pm 6,4 mg GAE.100 g-1, enquanto que Cieslik, Greda e Adamus (2006) apresentaram para o conteúdo fenólico da maçã, em peso seco, valores entre 905 e $1030 \mathrm{mg}$ GAE. $100 \mathrm{~g}^{-1}$.

\subsection{Determinação de flavanóis e atividade antioxidante}

A determinação de flavanóis no bagaço da maçã apresentou valores de 11,05 $\pm 1,24$ e 58,20 $\pm 6,56 \mathrm{mg} \mathrm{CE} 100 \mathrm{~g}^{-1}$ na amostra em base úmida e base seca, respectivamente (Tabela 1). Chinnici et al. (2004) encontraram valores totais de flavanóis (equivalente à catequina) de $223 \pm 26,4$ e 46,1 $\pm 9,22 \mathrm{mg} \cdot \mathrm{kg}^{-1} \mathrm{em}$ peso fresco, para maçã descascada e polpa de maçã, respectivamente. Esses valores são superiores aos encontrados em nosso estudo. Provavelmente a maior parte dos flavanóis foi extraída juntamente com o suco, restando no bagaço outras classes de flavonóides que se encontram mais fortemente ligados à parede celular, como os ácidos fenólicos e compostos fenólicos de alto peso molecular ligados a açúcares ou lignina.

Ainda na Tabela 1, podem ser vistos os valores da capacidade antioxidante determinada pelos métodos de seqüestro dos 
Tabela 1. Teores de flavanóis e atividade antioxidante (ABTS e DPPH) de extratos do bagaço de maçã cv. Gala safra 2006 obtidos com acetona $75 \%(\mathrm{v} / \mathrm{v})$.

\begin{tabular}{|c|c|c|c|c|c|}
\hline Amostras & Flavanóis $^{\mathrm{a}}\left(\mathrm{mg} .100 \mathrm{~g}^{-1}\right)$ & ABTS-TEAC $^{\mathrm{b}}\left(\mu \mathrm{mol}^{-1}{ }^{-1}\right)$ & ABTS-VCEAC ${ }^{c}\left(\mathrm{mg} .100 \mathrm{~g}^{-1}\right)$ & DPPH-TEAC $\left(\mu\right.$ mol.g $\left.^{-1}\right)$ & DPPH-VCEAC $\left(m g .100 \mathrm{~g}^{-1}\right)$ \\
\hline $\mathrm{BU}$ & $11,05 \pm 1,24$ & $4,83 \pm 0,30$ & $76,83 \pm 4,12$ & $7,43 \pm 0,43$ & $93,61 \pm 5,50$ \\
\hline BS & $58,20 \pm 6,56$ & $25,46 \pm 1,39$ & $404,68 \pm 1,70$ & $39,15 \pm 2,27$ & $493,06 \pm 8,91$ \\
\hline
\end{tabular}

Média $\pm \mathrm{DP}, \mathrm{n}=3 .{ }^{\mathrm{a}} \mathrm{CE}=$ equivalente à catequina; ${ }^{\mathrm{b}} \mathrm{TEAC}=$ atividade antioxidante equivalente ao Trolox em 7 minutos $(\mathrm{ABTS})$ e 30 minutos $(\mathrm{DPPH}) ;{ }^{\mathrm{c}} \mathrm{VCEAC}=$ atividade antioxidante equivalente à vitamina $\mathrm{C}$; $\mathrm{BU}=$ base úmida; e $\mathrm{BS}=$ base seca.

radicais $\mathrm{ABTS}^{\circ+}$ e DPPH para o bagaço de maçã cv. Gala. De acordo com essa tabela, o valor TEAC (ABTS) em base úmida foi de 4,83 $\pm 0,30 \mu \mathrm{mol}^{-1} \mathrm{~g}^{-1}$. Comparando os resultados obtidos com os descritos na literatura, pode-se constatar que Kuskoski (2003), avaliando a atividade antioxidante do fruto baguaçu (Eugenia Umbelliflora Berg) natural do Estado de Santa Catarina, Brasil, encontrou um TEAC de 3,08 $\mu \mathrm{mol.g}{ }^{-1}$ de fruto fresco e Sellappan, Akoh e Krewer (2002) constataram que os valores TEAC de mirtilo (Vaccinium myrtillos) e zarzamora (Rubus fruticosus) foram de 0,8 e 3,8 $\mu \mathrm{mol} . \mathrm{g}^{-1}$ de frutos frescos, respectivamente. Demonstrando assim que o bagaço de maçã cv. Gala contém uma elevada atividade antioxidante pelo ensaio de captura de radicais ABTS, sendo inclusive superior a algumas frutas usualmente consumidas.

Os valores VCEAC (ABTS) do bagaço de maçã cv. Gala foram de 404,68 $\pm 1,70$ por $100 \mathrm{~g}^{-1} \mathrm{em}$ base seca, superiores aos reportados por Kim et al. (2002) que encontraram valores de 205,4 $\pm 5,6 \mathrm{mg} .100 \mathrm{~g}^{-1} \mathrm{em}$ base seca VCEAC, para extratos dessa mesma variedade de maçã. Esses mesmos autores avaliaram extratos de outras variedades de maçãs produzidas em distintos países e encontraram valores que variaram de 205 a 559.mg. $100 \mathrm{~g}^{-1}$ em base seca.

Os valores TEAC (DPPH) da amostra analisada foram de 7,43 $\pm 0,43 \mu$ mol.g ${ }^{-1}$ em base úmida, valores próximos aos detectados por Villano et al. (2006) ao analisar 16 variedades de vinhos tintos produzidos no sul da Espanha, encontrando um valor médio de $9,44 \pm 3,97 \mu \mathrm{mol} \cdot \mathrm{mL}^{-1}$. Já o VCEAC (DPPH) foi de 93,61 $\pm 5,50 \mathrm{mg} \cdot 100 \mathrm{~g}^{-1}$ em base úmida e $493,06 \pm 8,91 \mathrm{mg} .100 \mathrm{~g}^{-1} \mathrm{em}$ base seca. Demonstrando, portanto, pelos dois métodos antioxidantes in vitro, utilizados neste estudo, que o bagaço de maçã cv. Gala possui uma atividade antioxidante expressiva, podendo ser aproveitado como fonte de antioxidantes naturais.

\subsection{Relação entre atividade antioxidante (TEAC) e conteúdo de fenólicos totais}

Utilizando-se o método ABTS neste estudo, o coeficiente de regressão entre o conteúdo de fenólicos totais e atividade antioxidante dos extratos obtidos a partir das diferentes concentrações de acetona foi $\mathrm{r}^{2}=0,9283, \mathrm{p}<0,05$ (Figura 2). Esse valor confirma os dados apresentados em diversos estudos, que mostram que a capacidade antioxidante é fortemente relacionada com o teor de compostos fenólicos totais presentes (MAKRIS; BOSKOU; ANDRIKOPOULOS, 2007; THAIPONG et al., 2006; ABDILLE et al., 2005; KAUR; KAPOOR, 2002). No estudo de Sun et al. (2002), foi encontrada uma relação direta $\left(\mathrm{r}^{2}=0,9788, \mathrm{p}<0,01\right)$ entre atividade antioxidante e o conteúdo de fenólicos totais em diferentes frutas.

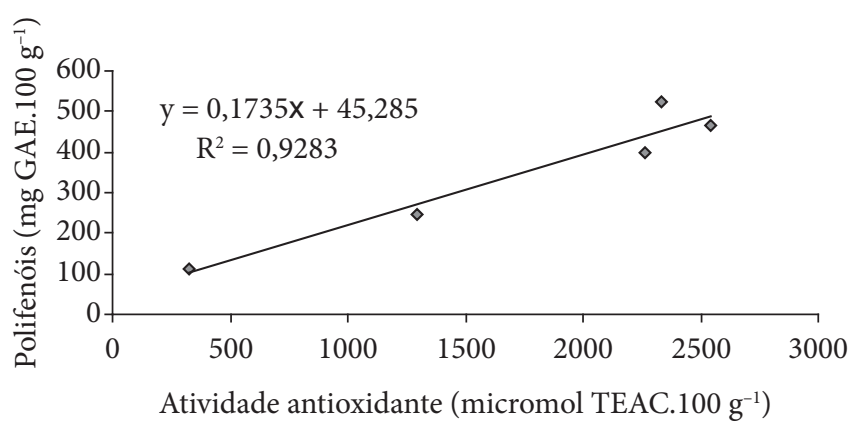

Figura 2. Correlação entre polifenóis totais dos extratos do bagaço de maçã cv. Gala em diferentes concentrações de acetona (0, 25, 50, 75 e $100 \%)$ com atividade antioxidante em TEAC $\left(\mu \mathrm{mol} . \mathrm{g}^{-1}\right)$, determinada pelo método ABTS; em base seca.

\subsection{Identificação dos ácidos fenólicos e avaliação da sua atividade antioxidante}

Para a extração das diferentes frações de ácidos fenólicos seguiu-se a metodologia descrita por Krygier, Sosuski e Hogge (1982), na qual se realiza uma extração inicial com tetrahidrofurano (THF) e se obtêm os ácidos fenólicos que se encontram livres (AFL) no vegetal. O resíduo dessa extração é novamente extraído com uma mistura de metanol/acetona/água e hidrolisado com $\mathrm{NaOH}$ para liberar os ésteres solúveis que se encontram esterificados com proteínas e/ou polipeptídeos, em seguida extraídos com uma mistura de éter etílico/acetato de etila/ THF obtendo-se a fração Ácidos Fenólicos Solúveis (AFES). O resíduo proveniente da extração dos AFES é hidrolisado e, em seguida, acidificado e extraído com uma mistura de éter etílico/ acetato de etila/THF, obtendo-se a fração de Ácidos Fenólicos Insolúveis (AFEI). Portanto são frações altamente purificadas.

Na Tabela 2, está apresentado o conteúdo de fenólicos totais e a atividade antioxidante das diferentes frações de ácidos fenólicos. De acordo com essa tabela, pode-se constatar que das três frações obtidas a AFES é mais rica em fenólicos totais $(260,01 \mathrm{mg}$ GAE. $100 \mathrm{~g} \mathrm{~g}^{-1}$ ), apresentando também maior capacidade em degradar os radicais ABTS $\left(57,36 \mu \mathrm{mol}^{-g^{-1}} \mu\right.$ molTEAC. $\left.{ }^{-1}\right)$.

A identificação e a quantificação dos ácidos fenólicos presentes nas frações AFL, AFES e AFEI presentes no bagaço de maçã cv. Gala podem ser observadas na Tabela 3. De acordo com essa tabela, pode-se constatar que o ácido salicílico está presente nas três frações avaliadas sempre em maior concentração; foram também identificados os ácidos gálico, propilgalato e sináptico em todas as frações. Na AFES, foram identificados dez diferentes ácidos fenólicos, sendo predominantes os ácidos salicílico 
Tabela 2. Atividade antioxidante (ABTS) e conteúdo de fenólicos totais das frações fenólicas do bagaço de maçã cv. Gala, safra 2005/2006.

\begin{tabular}{lccc}
\hline \multicolumn{2}{c}{$\begin{array}{c}\text { Fenólicos totais } \\
\text { mg GAE.100 g }\end{array}$} & \multicolumn{2}{c}{$\begin{array}{c}\text { ABTS (TEAC) } \\
\left(\mu \mathrm{mol}^{-1} \mathrm{~g}^{-1}\right)\end{array}$} \\
\hline AFL & 230,39 & AFL & 31,77 \\
AFES & 260,01 & AFES & 57,36 \\
AFEI & 208,90 & AFEI & 24,26 \\
\hline
\end{tabular}

$\overline{\mathrm{AFL}}$ = fração ácidos fenólicos livres; AFES = fração esterificada solúvel; $\mathrm{AFEI}=$ fração esterificada ligante insolúvel; ${ }^{1} \mathrm{GAE}$ : equivalente a ácido gálico; $\mathrm{e}^{2} \mathrm{TEAC}$ : atividade antioxidante equivalente ao Trolox.

Tabela 3. Conteúdo de ácidos fenólicos livres e de esterificados solúveis e insolúveis do bagaço de maçã cv. Gala safra 2005/2006.

\begin{tabular}{lccc}
\hline Ácidos fenólicos & AFL & AFES & AFEI \\
\hline Salićlico & $28,22 \pm 1,83$ & $52,77 \pm 4,50$ & $10,69 \pm 0,73$ \\
Cinâmico & nd & $0,084 \pm 0,01$ & $0,032 \pm 0,00$ \\
Vanílico & nd & $0,056 \pm 0,00$ & nd \\
p-cumárico & $0,070 \pm 0,01$ & $0,044 \pm 0,00$ & nd \\
Gálico & $0,065 \pm 0,01$ & $0,175 \pm 0,03$ & $0,075 \pm 0,01$ \\
Propilgalato & $0,097 \pm 0,01$ & $0,198 \pm 0,00$ & $0,039 \pm 0,00$ \\
Ferúlico & nd & $0,160 \pm 0,17$ & $0,079 \pm 0,04$ \\
Caféico & nd & $0,044 \pm 0,00$ & $0,033 \pm 0,03$ \\
Sináptico & $0,069 \pm 0,00$ & $0,140 \pm 0,04$ & $0,021 \pm 0,03$ \\
Elágico & nd & $0,051 \pm 0,01$ & nd \\
\hline
\end{tabular}

Resultados expressos em mg. $\mathrm{g}^{-1}$ de amostra seca. Média \pm desvio padrão, $\mathrm{n}=3$; $\mathrm{nd}$ = não detectado; AFL = fração ácidos fenólicos livres; AFES = fração esterificada solúvel; e AFEI = fração esterificada ligante insolúvel.

(52,77 mg. $\left.\mathrm{g}^{-1}\right)$, gálico $\left(0,175 \mathrm{mg} \cdot \mathrm{g}^{-1}\right)$, propilgalato $\left(0,198 \mathrm{mg} \cdot \mathrm{g}^{-1}\right)$, ferúlico $\left(0,160 \mathrm{mg} \cdot \mathrm{g}^{-1}\right)$ e sináptico $\left(0,140 \mathrm{mg} \cdot \mathrm{g}^{-1}\right)$, bem como traços de caféico, cinâmico e vanílico. Yeh e Yen (2006) cita que entre todos os ácidos hidrobenzóicos presentes nos vegetais, o ácido gálico é o que apresenta maior efetividade na inativação do radical ABTS. Estudos realizados por Hsieh, Yen e Chen (2005) e Meyer et al. (1998) observaram a propriedade do ácido ferúlico como seqüestrante de radicais livres, mesmo em concentrações muito reduzidas. Baggio et al. (2007) também constataram uma predominância de ácido salícilico na casca do café (Coffea arabica, L.). Já Lu e Foo (1997; 1999) identificaram nos extratos acetônicos de bagaço de maçã diferentes polifenóis, como ácido clorogênico, caféico e p-cumárico, além da floridzina, epicatequina e quercetina glicosídica.

\section{Conclusões}

Os resultados obtidos nas condições de realização desta pesquisa permitem inferir que: os diferentes sistemas de solventes utilizados na extração afetaram significativamente o conteúdo de fenólicos totais do bagaço de maçã e que o sistema solvente constituído por acetona $75 \%$ (v/v) em água foi o mais eficaz em extrair os compostos fenólicos totais; o bagaço de maçã cv. Gala apresentou elevada atividade antioxidante pelos dois métodos empregados; foram identificados os ácidos fenólicos salícilico, gálico, propilgalato e sináptico nas três frações avaliadas, o primeiro estando presente em maior concentração em todas as frações estudadas; os resultados obtidos indicam a possibilidade da utilização do bagaço de maçã cv. Gala como fonte de antioxidantes naturais.

\section{Agradecimentos}

Ao CNPq pelo auxílio financeiro.

\section{Referências bibliográficas}

ABIDILLE, M. D. H. et al. Antioxidant activity of the extracts from Dillenia indica fruits. Food Chemistry, v. 90, n. 4, p. 891-896, 2005.

ARNOUS, A.; MAKRIS, D.; KEFALAS, P. Correlation of pigment and flavanol content with antioxidant properties in selected aged regional wines form Greece. Journal of Food Composition and Analysis, v. 15, p. 655-665, 2002.

AOAC. ASSOCIATION OF OFFICIAL ANALYTICAL CHEMISTRY. Official methods of Analysis of the Association of Official Analytical Chemistry. 16 ed. Gaithersburg, Maryland, 1995. v. 2.

BAGGIO, J. et al. Identification of phenolic acids in coffee (Coffea arabica L.) dust and its antioxidant activity. Italian Journal of Food Science, v. 19, n. 2, p. 191-201, 2007.

BRAND-WILLIAMS, W.; CUVELIER, M. E.; BERSET, C. Use of free radical method to evaluate antioxidant activity. Lebensmittel Wissenschaft und -Technologie, v. 22, p. 25-30, 1995.

CIESLIK, E.; GREDA, A.; ADAMUS, W. Contents of polyphenols in fruits and vegetables. Food Chemistry, v. 94, p. 135-142, 2006.

CHINNICI, F. et al. Radical Scavenging Sctivities of Seels and Sulps from cv. Golden Delicious Apples as Related to their Phenolic Composition. Journal of Agricultural and Food Chemistry, v. 52, p. 4684-4684, 2004.

DRABROWSKI, K. J.; SOSULSKI, F. W. Quantification of free and hydrolysable phenolic acids in seeds by capillary gas-liquid chromatography. Journal of Agricultural and Food Chemistry, v. 32, p. 123-127, 1984.

EBERHARDT, M. V.; LEE, C. Y.; LIU, R. H. Antioxidant activity of fresh apples. Nature, v. 22, n. 405, p. 903-904, 2000.

GIADA, M. L. R.; MANCINI FILHO, J. Importância dos compostos fenólicos da dieta na promoção da saúde humana. Publicatio UEPG Ciencias Biologicas e da Saude, v. 12, n. 4, p. 7-15, 2006.

HEIM, K. E.; TAGLIAFERRO, A. R.; BOBILYA, D. J. Flavonoid antioxidants: chemistry, metabolism and structure-activity relationships. The Jounal of Nutritional Biochemistry, v. 13, p. 572-584, 2002.

HSIEH, C. L.; YEN, G. C.; CHEN, H. Y. J. Antioxidant activities of phenolic acids on ultraviolet radiation-induced erythrocyte and low density lipoprotein oxidation. Journal of Agricultural and Food Chemistry, v. 53, p. 6151-6155, 2005.

JEWELL, W. J.; CUMMINGS, R. J. Apple pomace energy and solids recovery. Journal of Food Science, v. 49, p. 407-410, 1984.

KAUR, C.; KAPOOR, H. C. Anti-oxidant activity and total phenolic content of some Asian vegetables. International Journal of Food Science Technology, v. 37, n. 2, p. 153-161, 2002.

KIM, D. O. et al. Vitamina C equivalente antioxidant capacity (VCEAC) of phenolics phytochemicals. Journal of Agricultural and Food Chemistry, v. 50, p. 3713-3717, 2002.

KIM, D. O.; JEONG, S. W.; LEE, C. Y. Antioxidant capacity of phenolic phytochemicals from various cultivars of plums. Food Chemistry, v. 81, p. 321-326, 2003.

KRYGIER, K.; SOSULSKI, F.; HOGGE, L. Free, esterified and insoluble-bound phenolic acids. Journal of Agricultural and Food Chemistry, v. 30, p. 334-336, 1982. 
KUSKOSKI, E. M. Caracterización de pigmentos em frutos de baguaçu (E. umbelliflora Berg). Sevilla, 2003. 230p. Tesis - (Doctorado Estudios Avanzados en Analisis Químico), Departamento de Alimetnos, Nutrición y Bromatologia, Universidad de Sevilla.

KUSKOSKI, E. M. et al. Actividad antioxidante de pigmentos antocianicos. Ciência e Tecnologia de Alimentos, v. 24, n. 4, p. 691-693, 2004

LOMMEN, A. et al. Application of directly coupled HPLC-NMR-MS to the identification and confirmation of quercetin glycosides and phloretin glycosides in apple peel. Analytical Chemistry, v. 72, p. 1793-1797, 2000.

LU, Y.; FOO, L. Y. Identification and quantification of major polyphenols in apple pomace. Food Chemistry, v. 59, p. 187-197, 1997.

LU, Y.; FOO, L. Y. Isolation and identification of procyanidins in apple pomace. Food Chemistry, v. 64, p. 511-518, 1999.

LU, Y.; FOO, L. Y. Antioxidant and radical scavengig activities of polyphenols from apple pomace. Food Chemistry, v. 68, p. 81-85, 2000.

MANACH, C. et al. Polyphenols: food sources and bioavailability. American Journal of Clinical Nutrition, v. 79, n. 5, p. 727-747, 2004.

MANACH, C.; MAZUR, A.; SCALBERT, A. Polyphenols and prevention of cardiovascular diseases. Current Opinion in Lipidology, v. 16, p. 77-84, 2005.

MAKRIS, D. P.; BOSKOU, G.; ANDRIKOPOULOS, N. K. Polyphenolic content and in vitro antioxidant characteristics of wine industry and other agri-food solid waste extracts. Journal of Food Composition and Analysis, v. 20, p. 125-132, 2007.

MEYER, A. S. et al. Fruit hidroxycinnamic acids inhibit human low density lipoprotein oxidation in vitro. Journal of Agricultural and Food Chemistry, v. 46, p. 1783-1787, 1998.

MOREIRA, A. V. B.; MANCINI FILHO, J. Atividade antioxidante das especiarias mostarda canela e erva-doce em sistema aquoso e lipídico. Nutrire, v. 25, p. 31- 46, 2003.

PESCHE, W. et al. Na industrial approach in the search of natural antioxidants from vegetable and fruit wastes. Food Chemistry, v. 97, p. 137-150, 2005.

RE, R. et al. Antioxidant activity applying an improved ABTS radical cation decolorization assay. Free Radical Biology and Medicine, v. 26, p. 1231-1237, 1999

RICE-EVANS, C.; MILLER, N. J.; PAPANGA, G. Structure-antioxidant activity relationships of flavonoids and phenolics acids. Free Radical Biology and Medicine, v. 20, p. 933-956, 1996.
ROSSI, J. A. J.; SINGLETON, V. L. Colorimetry of total phenolics with phosphomolybdic phosphotungstic acid reagents. American Journal of Enology and Viticulture, v. 16, p. 144-158, 1965.

SELLAPAN, S.; AKOH, C. C.; KREWER, G. Phenolic compounds and antioxidant capacity of Geogia-Grown blueberries and blackberries. Journal of Agricultural and Food Chemistry, v. 50, p.2432-2438, 2002.

SHOJI, T.; AKAZOME, Y.; IKEDA, K. M. The toxicology and safety of apple polyphenol extract. Food and Chemical Toxicology, v. 42, p. 959-967, 2004.

SOTERO, D. E. G. Caracterização química e avaliação da atividade antioxidante de frutos da Amazônia: chope (Gustavia augusta L.) sacha mangua (Grias neuberthii Macbr.) e macambo (Theobroma bicolor). São Paulo, 2002. Tese - (Doutorado em Ciência dos Alimentos), Curso de Pós-graduação em Ciência dos Alimentos, USP.

STRATIL, P.; KLEJDUS, B.; KUBÁN, V. Determination of phenolic compounds and their antioxidant activity in fruits and cereals. Talanta, v. 71, p. 1741-1751, 2007.

SUN, J. et al. Antioxidant and antiproliferative activities of common fruits. Journal of Agricultural and Food Chemistry, v. 50, p. 7449-7454, 2002.

THAIPONG, K. et al. Comparison of ABTS, DPPH, FRAP, and ORAC assays for estimating antioxidant activity from guava fruit extracts. Journal of Food Composition and Analysis, v. 19, p. 669-675, 2006.

TSAU, R. et al. Which poliphenol compounds contribute to the total antioxidant activities of apple. Journal of Agricultural and Food Chemistry, v. 53, n. 12, p. 4989-4995, 2005.

VALDERRAMA, P.; MARANFONI, F.; CLEMENTE, E. Efeito do tratamento térmico sobre a atividade de peroxidase (POD) e polifenoloxidase (PPO) em maçã (Mallus comunis). Ciência e Tecnologia de Alimentos, v. 21, n. 3, p. 321-325, 2001.

VILLAÑO, D. et al. Influence of enological practices on the antioxidant activity of wines. Food Chemistry, v. 95, p. 394-404, 2006.

WOLFE, K.; WU, X.; LIU, R. H. Antioxidant activity of apple peels. Journal of Agricultural and Food Chemistry, v. 53, p. 609-614, 2003.

YEH, C. T.; YEN, G. C. Effects of phenolics acids on human phenolsulfotransferases in relation to their antioxidant activity. Journal of Agricultural and Food Chemistry, v. 51, p. 1474-1479, 2006.

YILMAZ, Y; TOLEDO, R. T. Oxygen radical absorbance capacities of grape/wine industry byproducts and effect of solvent type on extraction of grape seed polyphenols. Journal of Food Composition and Analysis, v. 19, p. 41-48, 2006. 